\title{
Melting transition of two-dimensional crystals
}

\author{
A. Holz * \\ Institut für Theoretische Physik, Freie Universität Berlin, Arnimallee 3, 1 Berlin 33, Federal Republic of Germany
}

\author{
J. T. N. Medeiros
}

Instituto de Física, Universidade Fedẹral do Rio Grande do Sul, 90000 Porto Alegre, RS, Brazil

(Received 28 March 1977; revised manuscript received 9 August 1977)

\begin{abstract}
The solid-liquid phase transition (PT) in two-dimensional crystals is studied under the assumption that it is driven by the dissociation of elastic dipoles. It is shown that this PT is of first-order and corresponds to a dissociation transition of elastic dipoles. For systems where, via thermal nucleation metastable polycrystalline states are possible besides elastic-dipole formation, a more-complex phase diagram is postulated with lines of first- and second-order PT. In the light of these concepts molecular-dynamics experiments with long- and short-range interactions in two and three dimensions are discussed. Solid-liquid PT observed in various experimental monolayer systems and showing first- and second-order PT are interpreted in terms of the theory developed.
\end{abstract}

\section{INTRODUCTION}

We study in the following the break down of "long-range" order in two-dimensional (2D) crystals which can be considered as a solid-liquid phase transition (PT). This problem has been considered by Berezinskii, ${ }^{1}$ Kosterlitz and Thouless, ${ }^{2}$ and the analogous problem for Bose systems by Popov. ${ }^{3}$ It has been pointed out by Mermin ${ }^{4}$ that the $2 \mathrm{D}$ harmonic net has no long-range positional order at $T \neq 0$ due to the divergence of the displacement autocorrelation function $\langle[\overrightarrow{\mathrm{u}}(\overrightarrow{\mathrm{R}})$ $\left.\left.-\overrightarrow{\mathrm{u}}\left(\overrightarrow{\mathrm{R}}^{\prime}\right)\right]^{2}\right\rangle \sim \ln \left|\overrightarrow{\mathrm{R}}-\overrightarrow{\mathrm{R}}^{\prime}\right|$ for $\left|\overrightarrow{\mathrm{R}}-\overrightarrow{\mathrm{R}}^{\prime}\right| \rightarrow \infty$, where $u(\overrightarrow{\mathrm{R}})$ is the displacement from the assumed equilibrium position $\vec{R}$. However, if the lattice supports stable transverse phonons then the system exhibits directional long-range order

$$
\left.\left\langle\overrightarrow{\mathrm{r}}\left(\overrightarrow{\mathrm{R}}+\mathrm{a}_{1}\right)-\overrightarrow{\mathrm{r}}(\overrightarrow{\mathrm{R}})\right] \cdot\left[\overrightarrow{\mathrm{r}}\left(\overrightarrow{\mathrm{R}}^{\prime}+\overrightarrow{\mathrm{a}}_{1}\right)-\overrightarrow{\mathrm{r}}\left(\overrightarrow{\mathrm{R}}^{\prime}\right)\right]\right\rangle \rightarrow a_{1}^{2}
$$

for $\left|\vec{R}-\vec{R}^{\prime}\right| \rightarrow \infty$. Here $\vec{r}(\vec{R})$ is the instantaneous position of the lattice point $\vec{R}$ and $\vec{a}_{1}$ is a lattice unit vector. The vanishing of the long-range directional order or topological order is associated with a melting PT at $T_{c}\left(n_{d}\right)$, where $n_{d}$ is the density of elastic dipoles per unit area and will be explained later. At $T_{c}\left(n_{d}\right)$ the static shear modulus which is proportional to the order parameter of the crystalline phase vanishes together with part of the long-wavelength transverse phonon branch which represents the Goldstone mode of the ordered phase. This PT is observed to be in 3D systems always discontinuous, but in 2D systems first- and second-order melting PT have been observed. It is the objective of this paper to contribute to the clarification of the physical reasons for such behavior.

It has been pointed out in 1, 2, 3 that the destruction of topological order is associated with the dissociation of bound states of topological objects like pairs of dislocations forming elastic dipoles in the case of $2 \mathrm{D}$ crystals. Such ideas have been developed already earlier, e.g., by KuhlmannWilsdorf, ${ }^{5}$ for melting in 3D systems which she explains by means of a dislocation mechanism. In Sec. II of this paper a melting theory along similar lines is developed. Because the chemical potential for the thermal production of elastic dipoles is finite in contrast to the chemical potential of a single dislocation there exists always a finite concentration of elastic dipoles. With increasing $T$ the "dielastic" polarizability of these dipoles increases until it diverges at $T_{M}^{*}$. This implies that interactions between dipoles have to be taken into account even for dilute systems. Kosterlitz and Thouless ${ }^{2}$ study this interaction in the approximation that they take all interactions into account between one given dipole and all smaller dipoles being located in a circle as illustrated in Fig. 1(a). The dipoles located outside that circle of influence are ignored. We advocate the opposite approach in that we take only interactions between dipoles into account which do not overlap. This approach should be good as long as the density of dipoles $n_{d}$ is small compared to the number $z=1 / \pi\left\langle r^{2}\right\rangle$, where $\pi\left\langle r^{2}\right\rangle$ is the average area occupied by one dipole and $z$ thus roughly represents the number of "lattice sites" available per unit area for the dipole gas. It will be shown that the condition $n_{d} \ll z$ is satisfied over the whole crystalline domain. The interaction between the dipoles which is long ranged will be calculated in the Lorentz field approximation familiar from the theory of dielectric systems. This gives a condition on $n_{d}$ as a function of $T$ for a dissociation catastrophe to occur for the elastic dipole gas. It is shown that this theory is the analog to the Clausius-Mos- 

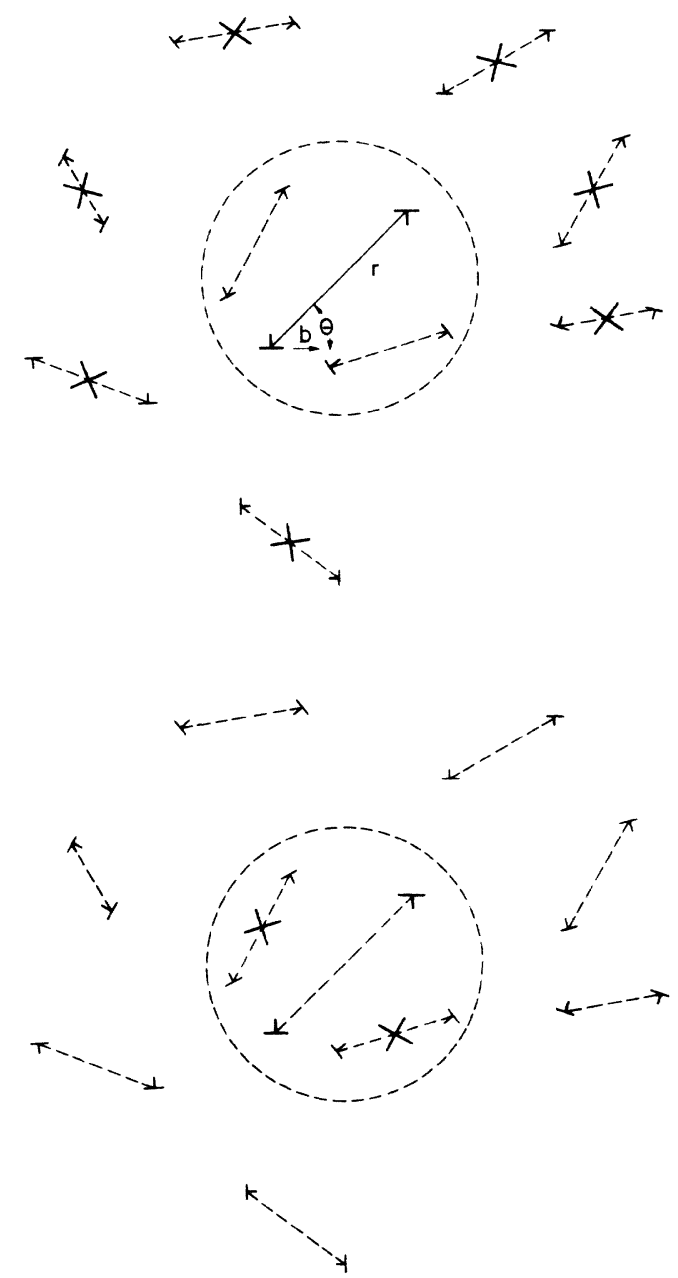

FIG. 1. Thickly drawn dipole is the reference dipole. The dashed circle separates the dipoles interacting with the reference dipole into two classes. The class of dipoles in (a) and (b) indicated by crosses is ignored. (a) corresponds to the work of Ref. 2 and (b) to the Lorentz field approach. The symbols $\overrightarrow{\mathrm{r}}, \overrightarrow{\mathrm{b}}$, and $\theta$ are explained in the text.

sotti theory for dielectric systems and has also to be considered as the first step in a systematic many-body theory for such systems. Due to the translational mobility of the elastic dipoles an equation of state for such objects taking the Van der Waals interaction between them into account has to be set up. This is done in Sec. III. It is shown there taking the second virial coefficient into account that the solid-liquid PT is of first order for $T\left(n_{d}\right)\left\langle T_{m}^{*}\right.$ and that the critical point $T_{C}$ $=T_{M}^{*}$ of the equation of state is located on the temperature axis and cannot be approached.

In Sec. IV we compare the theory with various molecular-dynamics computer experiments. There also second-order melting $\mathrm{PT}$ in $2 \mathrm{D}$ systems is observed. From the computer experiments of
Hockney and Brown ${ }^{6}$ where a polycrystalline phase is observed it follows that besides dislocation pairs $30^{\circ}$ crystalline boundaries forming metastable states have to be taken into account for triangular lattices when evaluating the free energy of the system. It is argued that this may lead to phase diagrams (PD) involving lines of first- and secondorder PT. In this context we develop a qualitative theory which explains why the PT observed for systems interacting with a long-range Coulomb interaction $1 / r$ changes from a second-order $\mathrm{PT}$ in $2 \mathrm{D}$ to a first-order $\mathrm{PT}$ in $3 \mathrm{D}$. In Sec. $\mathrm{V}$ the results are discussed in the light of a number of representative experiments on adsorbed monolayer systems, in particular $N_{2}$ on grafoil, ${ }^{7}$ butadiene iron tricarbonyl on graphite, ${ }^{8}$ and ${ }^{3} \mathrm{He}$ and ${ }^{4} \mathrm{He}$ monolayers on graphite. ${ }^{9}$ Some aspects of the theory have been presented elsewhere. ${ }^{10}$

\section{MEAN-FIELD THEORY OF ELASTIC DIPOLE SYSTEM}

We assume that with increasing $T$ dislocations appear in pairs ${ }^{1,2}$ forming elastic dipoles with zero resultant Burgers vector. The elastic dipoles dissociate at $T\left(n_{d}\right)$ which will be calculated in the mean-field approximation. This means that we take the polarization effect of neighboring elastic dipoles approximately into account as indicated in Fig. 1(b) whereas in Ref. 2 the polarization effect of the dipoles enclosed by a given dipole [see Fig. 1 (a) ] is taken into account. The dipoles neglected in each treatment are indicated in Figs. 1(a) and 1 (b) by big crosses. A rigorous treatment of the problem requires, of course, both effects to be considered. At the end of this section we will show that our approximation is reasonable.

For sake of simplicity we consider in the following an isotropic 2D crystal. Following Kosterlitz and Thouless ${ }^{2}$ we introduce a stress function $\chi(\overrightarrow{\mathrm{r}}),{ }^{11,12}$ which is related to the stress by

$$
\sigma_{i j}(\overrightarrow{\mathrm{r}})=\epsilon_{i k} \epsilon_{j l} \frac{\partial^{2} \chi(\overrightarrow{\mathrm{r}})}{\partial x_{k} \partial x_{l}}, \quad \epsilon=\left(\begin{array}{cc}
0 & 1 \\
-1 & 0
\end{array}\right) .
$$

Next one defines a source function $\eta(\vec{r})$ describing the distribution of dislocations and test sources and which obeys in an isotropic medium

$$
\nabla^{2} \nabla^{2} \chi(\overrightarrow{\mathrm{r}})=K \eta(\overrightarrow{\mathrm{r}}),
$$

where $K=4 \nu(\nu+\lambda) /(2 \nu+\lambda)$ and $\nu$ and $\lambda$ are Lamé coefficients. The solution of Eq. (2) can now be written in the form ${ }^{2}$

$$
\chi(\overrightarrow{\mathrm{r}})=K \int d^{2} r^{\prime} \eta\left(\overrightarrow{\mathrm{r}}^{\prime}\right) g\left(\overrightarrow{\mathrm{r}}-\overrightarrow{\mathrm{r}}^{\prime}\right),
$$

where

$$
g(\overrightarrow{\mathrm{r}}) \simeq(1 / 8 \pi) r^{2} \ln \left|r / r_{0}\right|, \quad r \gg a, g(0)=0 .
$$


Here $a$ and $r_{0}$ is the lattice constant and the hardcore radius of the dislocation, respectively. The contribution of the $\alpha$ th dislocation pair to $\chi(\vec{r})$ is

$\left.\chi^{\alpha}(\mathrm{r})=\frac{1}{2} K<\int d^{2} r^{\prime} \eta^{\alpha}\left(\overrightarrow{\mathrm{r}}^{\prime}\right) x_{i}^{\prime} x_{j}^{\prime}\right\rangle \frac{\partial^{2} g\left(\overrightarrow{\mathrm{r}}-\overrightarrow{\mathrm{r}}^{\alpha}\right)}{\partial x_{i}^{\alpha} \partial x_{j}^{\alpha}}$,

where

$\eta^{\alpha}(\overrightarrow{\mathrm{r}})=\epsilon_{i j} b_{j}^{\alpha}\left(\frac{\partial}{\partial x_{i}^{\alpha}} \delta^{2}\left(\overrightarrow{\mathrm{r}}-\overrightarrow{\mathrm{r}}^{\alpha}\right)-\frac{\partial}{\partial x_{i}^{\alpha}} \delta^{2}\left(\overrightarrow{\mathrm{r}}+\overrightarrow{\mathrm{r}}^{\alpha}\right)\right)$.

Here $\vec{b}^{\alpha}$ is the Burgers vector and the average \langle\rangle which involves a configurational and thermal average will be calculated in the single-molecule approximation where the test source field and the mean field of the surrounding elastic dipoles is taken into account in linear-response approximation. This leads to

$$
\begin{aligned}
\left\langle\int d^{2} r^{\prime}\right. & \left.\eta^{\alpha}\left(\overrightarrow{\mathrm{r}}^{\prime}\right) x_{i}^{\prime} x_{j}^{\prime}\right\rangle \\
= & \left\langle\int d^{2} r^{\prime} \eta^{\alpha}\left(\overrightarrow{\mathrm{r}}^{\prime}\right) x_{i}^{\prime} x_{j}^{\prime}\right. \\
& \left.\times e^{-\beta U_{\text {eff }}^{\left(r^{\prime}, \theta^{\prime}\right)}}\left(1+2 \beta b_{l}^{\alpha} \sigma_{l k}^{\alpha} \epsilon_{k s} x_{s}^{\alpha}\right)\right\rangle,
\end{aligned}
$$

where

$$
\begin{aligned}
& \sigma_{i j}^{\alpha}(\overrightarrow{\mathrm{r}})=\sigma_{i j}^{\prime}(\overrightarrow{\mathrm{r}})+\epsilon_{i k} \epsilon_{j l} \sum_{\alpha^{\prime} \neq \alpha} \frac{\partial^{2} \chi^{\alpha^{\prime}}(\overrightarrow{\mathrm{r}})}{\partial x_{k} \partial x_{l}}, \\
& U_{\text {elf }}(r, \theta)=\frac{b^{2} K}{4 \pi}\left(\ln \left|\frac{\overrightarrow{\mathrm{r}}}{r_{0}}\right|-\frac{1}{2} \cos 2 \theta\right)+2 \mu .
\end{aligned}
$$

Here the first term of Eq. (7) is due to the test source field and the second term results from the stress excerted by the surrounding dipoles. $U_{\text {eff }}$ (Ref. 12) is the internal energy of an elastic dipole where the two dislocations are a distance $r$ apart and $\theta$ is the angle between $\vec{r}$ and $\vec{b}$, as indicated in Fig. 1(a), and $2 \mu$ is the self-energy of an elementary dipole. Using the abbreviation

$$
P_{i j}^{\alpha} \equiv \frac{1}{2}\left\langle\int d^{2} r^{\prime} \eta^{\alpha}\left(\overrightarrow{\mathrm{r}}^{\prime}\right) x_{i}^{\prime} x_{i}^{\prime}\right\rangle,
$$

it is straightforward to derive a linear equation for the $P_{i j}^{\alpha}$ using Eqs. (6) and (7)

$$
\begin{aligned}
P_{i j}^{\alpha}= & \frac{\epsilon_{i k} \epsilon_{j l}\left[c_{1} \sigma_{k l}^{\prime}\left(\overrightarrow{\mathrm{r}}^{\alpha}\right)+c_{2} \delta_{k l} \sigma_{m m}^{\prime}\left(\overrightarrow{\mathrm{r}}^{\alpha}\right)\right]}{n_{d}(\overrightarrow{\mathrm{r}})} \\
& +K \sum_{\alpha^{\prime} \neq \alpha} \frac{P_{s q}^{\alpha}\left(c_{1} X_{s \alpha_{i j}}^{\alpha \alpha^{\prime}}+c_{2} X_{s \alpha_{i j p}}^{\alpha \alpha^{\prime}}\right)}{n_{d}(\overrightarrow{\mathrm{r}})}
\end{aligned}
$$

where

$$
X_{s q ; i j}^{\alpha \alpha^{\prime}} \equiv \frac{\partial^{4} g\left(\overrightarrow{\mathrm{r}}^{\alpha}-\overrightarrow{\mathrm{r}}^{\alpha^{\prime}}\right)}{\partial x_{s}^{\alpha^{\prime}} \partial x_{a}^{\alpha^{\prime}} \partial x_{i}^{\alpha^{\prime}} \partial x_{j}^{\alpha^{\prime}}}
$$

and

$$
c_{1}=-2 \beta b^{2} n_{d}(\mathrm{r})\left\langle r^{2}\right\rangle, \quad c_{2}=\beta b^{2} n_{d}(r)\left\langle r^{2} \cos 2 \theta\right\rangle .
$$

Here $b^{2}=\left(\overrightarrow{\mathrm{b}}^{\alpha}\right)^{2}$, and the density of dipoles $n_{d}(\overrightarrow{\mathrm{r}})$ per unit area has been introduced. The averages \langle\rangle are calculated using Eq. (8), and Einstein summation convention is used. For isotropic distribution of dipoles one can handle Eq. $\left(6^{\prime}\right)$ in the Lorentz field approximation which yields

$$
\begin{aligned}
P_{i j}= & \epsilon_{i k} \epsilon_{j l}\left[c_{1} \sigma_{k l}^{\prime}(r)+c_{2} \delta_{k l} \sigma_{m m}^{\prime}(r)\right]+4 K c_{1}\left[4 P_{12}\left(1-\delta_{i j}\right) A_{12,12}+\left(P_{11}+P_{22}\right) \delta_{i j} A_{i j}\right] \\
& -\frac{K n_{d}(r)}{4}\left[\left(1-\delta_{i j}\right) c_{1} P_{12}+\delta_{i j}\left(P_{11}+P_{22}\right)\left(c_{1}+c_{2}\right)\right]
\end{aligned}
$$

where $P_{i j} \equiv P_{i j}(\overrightarrow{\mathrm{r}})=P_{i j}^{\alpha} n_{d}(\overrightarrow{\mathrm{r}})$, and a uniform polarization of the dipoles has been assumed. Let us point out that in the following no use of the $\overrightarrow{\mathbf{r}}$ dependence of $n_{d}(\overrightarrow{\mathrm{r}})$ will be made. In any case only $n_{d}(\vec{r})$ distributions are admitted which vary on a scale macroscopic in comparison to the extension of the Lorentz circle. The second term in Eq. (9) originates from summation within the Lorentz circle which does not vanish even in the isotropic case. The following abbreviations have been used in Eq. (9):

$$
A_{12,12} \equiv \sum_{\alpha^{\prime} \neq 0}^{\prime} X_{11,12}^{0 \alpha^{\prime}}, \quad A_{i j} \equiv \sum_{\alpha^{\prime} \neq 0}^{\prime} X_{p p, i j}^{0 \alpha^{\prime}},
$$

where the prime indicates that summation is performed over the dipoles located in the first octant. The third term in Eq. (9) is due to the continuous integration outside the Lorentz circle which can be expressed in terms of a contour integral over this circle. Depolarization effects due to the surface of the system, i.e., the contour enclosing the whole system are contained in the first term of Eq. (9) where the stress field inside the medium is used. The solution of Eq. (9) is easily obtained and using Eqs. (3) and (5), $\chi(\overrightarrow{\mathrm{r}})$ can be expressed in the form

$$
\begin{aligned}
\chi(\overrightarrow{\mathrm{r}}) \simeq & K \int d^{2} r^{\prime} \eta\left(\overrightarrow{\mathrm{r}}^{\prime}\right) g\left(\overrightarrow{\mathrm{r}}-\overrightarrow{\mathrm{r}}^{\prime}\right) \\
& +K \int d^{2} r^{\prime} P_{i j}\left(\overrightarrow{\mathrm{r}}^{\prime}\right) \frac{\partial^{2} g\left(\overrightarrow{\mathrm{r}}-\overrightarrow{\mathrm{r}}^{\prime}\right)}{\partial x_{i}^{\prime} \partial x_{j}^{\prime}} .
\end{aligned}
$$

Here $\eta(\overrightarrow{\mathbf{r}})$ is the test source function inside the medium and $P_{i f}(\overrightarrow{\mathrm{r}})$ is a linear function of the stress $\left\{\sigma_{i j}^{\prime}\right\}$ produced by the test sources inside the med- 
ium as follows from the linear inhomogeneous Eq. (9). Using

$$
\sigma_{i j}^{\prime}(\overrightarrow{\mathrm{r}})=\epsilon_{i k} \epsilon_{j l} \frac{\partial^{2} \chi(\overrightarrow{\mathrm{r}})}{\partial x_{k} \partial x_{i}},
$$

one can solve the linear inhomogeneous integral
Eq. (10) by means of Fourier transformation and obtains the stress function $\chi(\vec{r})$ in the form

$$
\chi(\overrightarrow{\mathbf{r}})=K^{\prime} \int d^{2} r^{\prime} \eta\left(\overrightarrow{\mathbf{r}}^{\prime}\right) g^{m}\left(\overrightarrow{\mathbf{r}}-\overrightarrow{\mathbf{r}}^{\prime}\right),
$$

where

$$
g^{m}(\overrightarrow{\mathrm{r}})=\frac{1}{(2 \pi)^{2}} \int d^{2} k e^{i \overrightarrow{\mathrm{k}} \cdot \overrightarrow{\mathrm{r}}} \frac{g_{\overrightarrow{\mathrm{k}}}}{1+2\left(c_{1} K\right)^{2}\left(K^{\prime} / K\right)\left(\alpha^{\prime} f_{1, \overrightarrow{\mathrm{k}}}+\beta^{\prime} f_{2, \overrightarrow{\mathrm{k}}}\right)} .
$$

Here $g_{\overrightarrow{\mathrm{k}}}, f_{1, \overrightarrow{\mathrm{k}}}$, and $f_{2, \overrightarrow{\mathrm{k}}}$ are the Fourier transforms of $g(\overrightarrow{\mathrm{r}}), X_{11 ; 22}^{0 \alpha}$, and $X_{22 ; 22}^{0 \alpha}-X_{11 ; 11}^{0 \alpha}$, respectively, where one obtains using Eq. (4)

$$
\begin{aligned}
& X_{11 ; 22}^{0 \alpha}=\left(1 / 2 \pi r^{2}\right)\left(8 x_{1}^{02} x_{2}^{02}-1\right), \\
& X_{22 ; 22}^{0 \alpha}-X_{11 ; 11}^{0 \alpha}=\left(1 / \pi r^{2}\right)\left(x_{1}^{02}-x_{2}^{02}\right),
\end{aligned}
$$

and $\overrightarrow{\mathrm{r}}^{\alpha}=r\left(x_{1}^{0}, x_{2}^{0}\right)$. Furthermore we have introduced the abbreviation

$$
\begin{aligned}
K^{\prime} & \equiv K /\left(1-\frac{K\left(c_{1}+c_{2}\right)\left(1+\frac{1}{4} K c_{1}\right)}{1+\frac{1}{2} K\left(c_{1}+c_{2}\right)}\right), \\
\alpha^{\prime} & \equiv\left[K c_{1}+\left(\frac{1}{4}-16 A_{12 ; 12} / n_{d}\right)^{-1}\right]^{-1}, \\
\beta^{\prime} & \equiv\left(2 A_{11} / n_{d}\right) /\left[1+K\left(\frac{1}{2} c_{1}+c_{2}\right)\right],
\end{aligned}
$$

where $\alpha^{\prime}$ and $\beta^{\prime}$ are dimensionless. It follows from Eq. (12) that the presence of the elastic dipoles changes $K$ into $K^{\prime}$. Because this quantity is proportional to the static shear modulus the denominator of Eq. (15) plays the role of a dielectric function ${ }^{2}$ for that problem. Its divergence for $c_{1}+c_{2}=-2 / K$ signals the dissociation of the elastic dipoles and $\mathrm{K}^{\prime} \rightarrow 0^{+}$implies that the long-wavelength shear modulus vanishes.

Let us point out that $g^{m}\left(\overrightarrow{\mathrm{r}}-\overrightarrow{\mathrm{r}}^{\prime}\right)$ can be evaluated by means of contour integration. That it differs from $g\left(\overrightarrow{\mathbf{r}}-\overrightarrow{\mathbf{r}}^{\prime}\right)$ is due to the tensor character of the stress field of the elastic dipoles. Because for $K^{\prime} \rightarrow 0^{+}, \alpha^{\prime}$ and $\beta^{\prime}$ remain finite, it follows that $g^{m}(\overrightarrow{\mathrm{r}}) \rightarrow g(r)$ and we can restrict our discussion to $K^{\prime}$. That $\alpha^{\prime}$ and $\beta^{\prime}$ remain finite for $K^{\prime} \rightarrow 0$ can be shown as follows. From Eq. (8) together with the definition of $c_{1}$ and $c_{2}$ it follows that $c_{1}<0$ and $c_{2}>0$ holds. The denominator of Eq. (17) approaches $\frac{1}{2} K c_{2}>0$ for $K^{\prime} \rightarrow 0^{+}$. From the definition of $A_{12 ; 12}$ and Eq. (14a) follows that $A_{12 ; 12} / n_{d} \ll 1$ because for a homogeneous distribution of dipoles $A_{12 ; 12} \equiv 0$ and the discrete summation will not modify that result too much. Setting $A_{12 ; 12} \equiv 0$ in Eq. (16) and noting that $\left|c_{2}\right| \ll\left|c_{1}\right|$ shows that $\alpha^{\prime}$ remains finite for $K^{\prime} \rightarrow 0$. It follows from this that the essential properties of the system can be discussed in terms of $K^{\prime}$ only.

Let us point out that Eq. (15) has been derived within the Lorentz-field approximation. The "di- elastic" constant defined by Eq. (15)

$$
\epsilon_{e}=1-\frac{K\left(c_{1}+c_{2}\right)\left(1+\frac{1}{4} K c_{1}\right)}{1+\frac{1}{2} K\left(c_{1}+c_{2}\right)}
$$

is therefore to be considered as analogous to the dielectric constant given by the Clausius-Mossotti relation for a 2D Coulomb gas problem. This relation is given by

$$
(\epsilon-1) /(\epsilon+1)=\pi n \alpha_{M}(T)[1+S(n, T)] .
$$

Here $\alpha_{M}$ is the electronic polarizability and $S(n, T)$ (Ref. 13) represents a virial expansion in the density $n$ of paired charges when one goes beyond the Lorentz-field approximation. In a similar fashion Eq. (18) represents the first step to a systematic solution of Eq. (6) as an expansion in the density $n_{d}$ of elastic dipoles. One can improve on this result on setting up a density expansion for the solution to Eq. $\left(6^{\prime}\right)$ as has been done in Ref. 13 for dielectric problems. To first order in $n_{d}$ one obtains an improvement of Eq. (18) by substituting in Eq. (18), $c_{1,2}-c_{1,2}\left[1+S\left(n_{d}, T\right)\right]$, where

$$
S\left(n_{d}, T\right)=n_{d} B_{2} K^{2} c_{1}^{2}\left(g_{1}+g_{2} c_{2} / c_{1}\right) / n_{d}^{2}+O\left(n_{d}^{2}\right)
$$

and $g_{1}>0, g_{2}>0$ are numerical factors of $O(1)$. Here

$$
B_{2}=\frac{1}{8 \pi} \int_{0}^{\infty} d r \frac{r^{-3} n(r)}{n_{d}^{2}} \sim \frac{1}{8 \pi} \int_{0}^{\infty} d r r^{-3} e^{-\beta \phi(r)},
$$

where $n(\vec{r})$ is the pair distribution function for two elastic dipoles and the standard approximation expressing $n(\vec{r})$ in terms of the pair potential $\phi(r)$ has been made.

The instability condition $K^{\prime}=0$ within the present approximation can be put into the form

$$
\frac{1}{2} K \beta b^{2} n_{d}\left[1+S\left(n_{d}, T\right)\right]\left(\left\langle r^{2}\right\rangle-\frac{1}{2}\left\langle r^{2} \cos 2 \theta\right\rangle\right)=1 .
$$

It follows from this that the perturbation theory for the calculation of $S\left(n_{d}, T\right)=B_{2}^{\prime} n_{d}+C_{2}^{\prime} n_{d}^{2}+O\left(n_{d}^{3}\right)$ can be patterned according to the theory developed by De Boer et al. ${ }^{13}$ We have to point out, however, that we did not prove that the general expression for the instability condition $K^{\prime}=0$ has to have the form of Eq. (20). The evaluation of $B_{2}^{\prime}$ and $C_{2}^{\prime}$ 


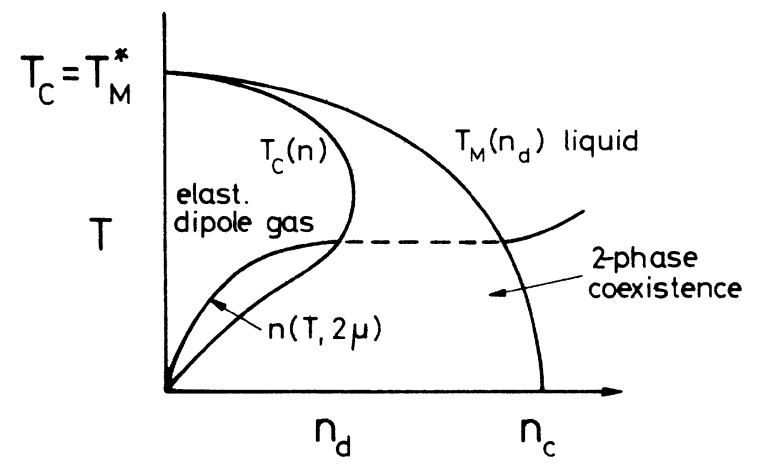

FIG. 2. Phase diagram showing solid-liquid PT mediated by first-order elastic dipole gas-dislocation plasma PT.

requires the knowledge of the two-and three-point dipole distribution function. This problem will be considered later.

For a discussion of Eq. (20) assume first that we set $S\left(n_{d}, T\right)=0$. Then Eq. (20) allows two solutions in $T$. The low $T$ solution which is associated with the ordering of elastic dipoles is an artifact of the static treatment of the problem and neglect of activation energies due to the discrete lattice. The high- $T$ solution defines for $n_{d} \rightarrow 0$ a critical temperature $T_{N}^{*}=\left(b^{2} K / 4 \pi\right) / 4 k_{B}$ which is obtained from $\left\langle r^{2}\right\rangle \rightarrow \infty$, and for $n_{d} \neq 0$ one obtains $T_{M}\left(n_{d}\right)$ $<T *$. In Fig. 2 we have drawn the dissociation temperature $T_{M}\left(n_{d}\right)$ connecting the points $T_{M}^{*}$ and $n_{c}$.

Let us consider next the range of applicability of Eq. (20). In connection with this problem it is very helpful to have some knowledge of the theory of dielectrics which has been reviewed by Brown. ${ }^{14}$ The analogous problem there is the problem of interaction of polar and polarizable molecules. The polar part of the elastic dipole corresponds to its elastic dipolemoment in its ground state, i.e., where $U_{\text {eff }} \sim 2 \mu$ and its extension is of the order of the hard core radius $r_{0}$ of the dislocation. The polarizable part of the dipole moment results from its possibility to assume extensions large compared to $r_{0}$. It has been pointed out by Onsager that the Lorentz-field approximation may be a good method for nonpolar molecules, i.e., polarizable molecules without permanent moment. For polar molecules, however, there is no reason to expect it to be a good approximation. It is just the spurious low-temperature solution of Eq. (20) which corresponds to an ordering transition for the elastic dipoles which must be dismissed on grounds of Onsagers objection. In order to treat the verylow- $T$ problem where the polar part of the elastic dipoles is dominating Onsager's reaction field method $^{15}$ has to be used. Because at low $T$ additional problems due to activation of dislocation motion arise and because it can be assumed that the melting $\mathrm{PT}$ is driven by the dissociation of the elastic dipoles, an effect due to the polarizability, we ignore that problem from now on. Consequently the Lorentz-field method leading to Eqs. (12) and $(20)$ can be considered as a first step in a systematic approach to calculate the elastic properties of the system. In order to improve the method $S\left(n_{d}, T\right)$ should be calculated. It has been pointed out that the correct computation of $B_{2}^{\prime}$ and $C_{2}^{\prime}$ requires the knowledge of the two- and threepoint elastic dipole distribution function. The problem now is, however, that due to the mobility of the elastic dipoles and their mutual attraction via Van der Waals forces such correlation functions have to be calculated self-consistently. Accordingly further instabilities may arise in the system, e.g., gas-liquid-type PT which require special treatment. This problem will be studied in Sec. III.

Finally we have to show that in the whole domain to the left of the curve $T_{M}\left(n_{d}\right)$ in Fig. 2 the assumption holds on which the Lorentz-field approximation is based, namely, that overlap effects between neighboring elastic dipoles can be neglected. For sake of simplicity we will demonstrate this by neglecting the term $S\left(n_{d}, T\right)$ and the term $\left\langle r^{2} \cos 2 \theta\right\rangle$ in Eq. (20) which is certainly less important than $\left\langle r^{2}\right\rangle$. Approximately then Eq. (20) can be put into the form

$$
n_{d} / \pi\left\langle r^{2}\right\rangle=\frac{1}{8}\left(T / T_{M}^{*}\right)
$$

Because $1 / \pi\left\langle r^{2}\right\rangle=z$ roughly defines the number of sites available for $n_{d}$ dipoles per unit area we get

$$
n_{d} / z=\frac{1}{8}\left(T / T_{M}^{*}\right),
$$

where the low $-T$ behavior is not correctly described as has been explained earlier. It follows that each eighth "site" is occupied at $T=T_{M}^{*}$ and even less for $T<T_{M}^{*}$. This implies that the dissociation catastrophe occurs at densities where rather weak overlap exists. Accordingly it is justified to a certain extent to neglect overlap effects in the first approximation. We like to point out that this is a rather common feature of such problems and is also observed in the insulator-metal PT which arises over a polarization catastrophe. ${ }^{16}$

\section{VAN DER WAALS THEORY FOR ELASTIC DIPOLE INTERACTION}

Due to the polarizability of the elastic dipoles they interact with each other via a Van der Waals interaction. The interaction energy of the $\alpha$ th elastic dipole in the strainfield $\sigma^{\alpha^{\prime}}$ of the $\alpha^{\prime}$ th dipole is given by

$$
\phi^{d-d}=2\left(\overrightarrow{\mathrm{b}}^{\alpha} \cdot \sigma^{\alpha^{\prime}} \cdot \overrightarrow{\mathrm{x}}^{\alpha}\right) .
$$


Here $\left(x_{1}^{\alpha}, x_{2}^{\alpha}\right) \equiv r^{\alpha}\left(-\sin \theta_{\alpha}, \cos \theta_{\alpha}\right)$, where $r^{\alpha}$ gives the extension of the dipole and $\theta_{\alpha}$ is the angle between $\overrightarrow{\mathrm{r}}^{\alpha}$ and $\overrightarrow{\mathrm{b}}^{\alpha}$. The thermal average of Eq. (21) can now be calculated in linear-response theory leading to

$$
\phi_{\mathrm{av}}^{d d}=-4 \beta\left\langle\left(b_{i}^{\alpha} \sigma_{i j}^{\alpha \prime} \epsilon_{j s} x_{s}^{\alpha}\right)^{2}\right\rangle .
$$

The average in Eq. (22) is computed with respect to $\exp \left[-\beta\left(U_{\text {eff }}^{\alpha}+U_{\text {eff }}^{\alpha^{\prime}}\right)\right]$, where $U_{\text {eff }}$ is defined in Eq. (8). In order to evaluate Eq. (22) one expresses $\sigma^{\alpha^{\prime}}$ by means of Eq. (1), where $\chi^{\alpha^{\prime}}(\overrightarrow{\mathrm{r}})$ can be calculated from Eq. (3) by using Eq. (5b). Partial integration allows then to express $\chi^{\alpha^{\prime}}(\overrightarrow{\mathrm{r}})$ in the form

$$
\begin{aligned}
\chi^{\alpha^{\prime}}(\overrightarrow{\mathrm{r}})=K\left[\epsilon_{i j} b_{i}^{\alpha^{\prime}}\right. & \left(\frac{\partial}{\partial x_{i, \alpha^{\prime}}} g\left(\overrightarrow{\mathrm{r}}-\overrightarrow{\mathrm{r}}^{\alpha^{\prime}}\right)\right. \\
& \left.\left.-\frac{\partial}{\partial x_{i, \alpha^{\prime}}} g\left(\mathrm{r}+\mathrm{r}^{\alpha^{\prime}}\right)\right)\right] .
\end{aligned}
$$

From Eq. (23) one obtains using Eq. (1)

$$
\sigma_{i j}^{\alpha^{\prime}}=2 K \epsilon_{i k} \epsilon_{j l} \epsilon_{s q} b_{q}^{\alpha^{\prime}} x_{p}^{\alpha^{\prime}} \frac{\partial^{4} g(\overrightarrow{\mathrm{r}})}{\partial x_{k} \partial x_{l} \partial x_{s} \partial x_{p}} .
$$

Equation (24) has to be inserted into Eq. (22) and one obtains after some simple but tedious algebra

$$
\phi_{\mathrm{ar}}^{d-a}=-\beta\left(\frac{K}{4 \pi}\right)^{2} \frac{b^{4}}{R^{4}}\left(\left\langle r^{2}\right\rangle-\frac{1}{2}\left\langle r^{2} \cos 2 \theta\right\rangle\right)^{2},
$$

where $R$ is the distance between the two dipoles. It follows from Eq. (25) by comparison with Eq. (20) that the factor $\left\langle r^{2}\right\rangle-\frac{1}{2}\left\langle r^{2} \cos 2 \theta\right\rangle$ enters both formulas in the same fashion. The interaction Eq. (25) diverges therefore also at the critical temperature $T_{M}^{*}$. The low-T divergence of Eq. (25) is due to the same reasons as the low $-T$ divergence of Eq. (20) and has to be considered as an artifact of the method. Because we are not interested in the low $T$ properties of the system and at higher $T$ the main $T$-dependence is due to the term involving the thermal average in Eq. (22) the low- $T$ divergence will be ignored from now on.

Due to the attractive nature of the Van der Waals interaction and particularly due to its divergence for $T \rightarrow T_{M}^{*}$ a gas-liquid-type PT of the elastic dipole system can be expected. A reasonable approximation to obtain a physical idea of the properties of that system is to set up a Van der Waals equation of state. In order to calculate the second virial coefficient of the equation of state the shortrange interaction of two elastic dipoles has to be known. The point here is that the composite object formed by two elastic dipoles must have a positive ground-state energy because it also represents a metastable state. If that would not be true the ground state of the crystal would have never been stable at all and disintegrated via the formation of such objects. For sake of simplicity we assume in the following that the repulsive interaction which originates from the above effect can be represented by a hard core repulsive potential. The hard core is introduced essentially for computational reasons and should be of the order of $\left\langle r^{2}\right\rangle^{1 / 2}$, i.e., of the order of the distance where Eq. (25) breaks down. We assume that the hard-core radius $r_{\mathrm{hc}}$ is given by $r_{\mathrm{hc}}^{2}=\gamma \sigma^{2}=\gamma\left(\left\langle r^{2}\right\rangle\right.$ $\left.-\frac{1}{2}\left\langle r^{2} \cos 2 \theta\right\rangle\right)$, where $\gamma$ is a factor of $O(1)$ and will be determined later. The second virial coefficient $B$ can then be written in the standard form

$$
B=n_{d}\left(b_{v}-a_{v} / k_{B} T\right),
$$

where, however, $a_{v}$ and $b_{v}$ are $T$ dependent and given by

$$
a_{v}=\frac{\pi}{3 \gamma}\left(\frac{K}{4 \pi}\right)^{2} \frac{b^{4}}{k_{B} T} \sigma^{2}, \quad b_{v}=\frac{1}{2} \pi \gamma \sigma^{2} .
$$

The Van der Waals equation of state reads now

$$
\left(p_{d}+a_{v} / v_{d}^{2}\right)\left(v_{d}-b_{v}\right)=k_{B} T .
$$

Here $p_{d}$ is the pressure exerted by the dipoles and $v_{d}$ is the specific area per dipole. In the present approximation where the harmonic lattice does not interact with the dislocations the total pressure is obtained by adding $p_{d}$ to the pressure exerted by the harmonic lattice. It is well known that Eq. (27) shows gas-liquid PT of first order with a critical point $T_{c}$ defined by the solution of

$$
k_{B} T_{c}=\left(\frac{8}{27}\right) a_{v}\left(T_{c}\right) / b_{v}\left(T_{c}\right) \text {. }
$$

Inserting $a_{v}$ and $b_{v}$ from Eq. (26b) into Eq. (28) one obtains

$$
\frac{k_{B} T_{c}}{(1 / 4 \pi) K b^{2}}=\frac{4}{9 \gamma} \text {. }
$$

At this stage one has to remember that our theory makes sense only as long as the elastic dipoles are not dissociated thermally, i.e., for $T \leq T_{M}^{*}$. If we identify for the time being $T_{c}$ with $T_{M}^{*}$ then we obtain from Eq. (29), $\gamma=\frac{16}{9}$. This leads to a hard-core radius $r_{\mathrm{hc}}=\frac{4}{3} \sigma$. In case that the region $\pi \sigma^{2}$ would have been inpenetrable then $\gamma=4$ should have been obtained. We have obtained thus a rather reasonable result considering the rather simple theory which has been used. It is, however, obvious that the fitting of $\gamma$ such that $T_{c}=T_{M}^{*}$ results does not justify the assumption that $T_{c}=T_{M}^{*}$ has to hold in the first place. That this is suggestive on more general grounds is explained presently.

The theory is based on the following idea. Two elastic dipoles attract each other when they are far away compared to $\left\langle r^{2}\right\rangle$, but repel each other when they come too close. The long-range Van 
der Waals attraction is a well-established phenomena and needs not be proven further. The shortrange repulsion results from the fact that the Van der Waals attraction must be bounded from below due to the assumed stability of the "crystalline" ground state. Long-range attraction and shortrange repulsion and in addition well identifiable objects as demonstrated at the end of Sec. II are the necessary requisites to set up a gas-liquid theory. Because with increasing $T$ the Van der Waals attraction Eq. (25) increases and approaches infinity at $T^{*}$ it follows that the gas-liquid coexistence curve in contrast to what happens in $T$-independent interacting systems should bend back to the $T$ axis. Because approaching $T_{M}^{*}$ the polarizability of one single elastic dipole gets infinity continuously, but then stays infinite we can assume that also in the case that the concentration $n_{d}$ approaches zero this point marks a continuous PT. We postulate therefore that the critical quantities are $T_{c}=T_{m}^{*}, p_{c}=0$, and $v_{c}=\infty$. Because the coexistence curve labeled $T_{c}\left(n_{d}\right)$ in Fig. 2 connects the origin with $T_{M}^{*}$ whereas the curve $T_{M}\left(n_{d}\right)$ which represents the dissociation catastrophe line goes from $n_{c}$ to $T_{M}^{*}$ it is plausible to postulate that the region between both curves is a two phase coexistence region. Let us point out that the concentration $n_{d}=n_{c}$ defined by $T_{M}\left(n_{c}\right)=0$ may be obtained via classical equation of motion techniques but is not needed in the following. On account of the above argumentation we may assume that the liquid phase is not a liquid of elastic dipoles but a plasma of unbounded dislocations. Accordingly the PT leads into the same plasma state as the dissociation catastrophe. This implies that the equation of state Eq. (27) applies to the region of the elastic dipole gas in Fig. 2 and its instability into a coalesced state, but not to the plasma phase. Because the coalesced phase of elastic dipoles gets into the range of density where the dissociation catastrophe occurs a transition into the plasma phase occurs; without that the system recovers in the liquid dipolar phase. It would be too difficult to take that effect in Eq. (27) into account as it would be too difficult to take the gasliquid instability in Eq. (20) into account. This is as a matter of fact not necessary because one knows that the gas-liquid PT at low $T$ leads to densities which are of the order of magnitude of the repulsive core region. Because $n_{c}$ is much less than this no intermediate liquid dipolar phase should arise.

Let us point out that the given variable in the present problem is the chemical potential $2 \mu$ defined by $U_{\text {eff }}$ in Eq. (8). The density $n_{d}$ of elastic dipoles has therefore to be obtained via the equation of state Eq. (27) and the relation $2 \mu$ $=\int{ }^{p} d v_{d}(p) d p$. In Fig. 2 we have drawn schematically $n=n(T, 2 \mu)$. According to this theory the solidliquid PT is always of first order. The apparent decrease of entropy during the gas-liquid PT is compensated by the increase of entropy due to dissociation of the elastic dipoles which occurs simultaneously. Accordingly one does not have the paradoxical result that the destruction of crystalline order is associated with a net decrease of entropy.

Finally we would like to point out that the "dielastic" constant $\epsilon_{e}$ defined by Eq. (18) also allows to determine the transversal shear mode velocity by means of $c_{t}^{\prime}=c_{t} / \epsilon_{e}$. Here $c_{t}$ is the shear mode velocity when no dislocations are present. In the liquid state one has $\epsilon_{e}=\infty$ and this leads to a vanishing of part of the transversal phonon branch. In principle the present theory could be extended into the liquid state where a $q$-dependent "dielastic" constant $\epsilon_{\alpha}$ had to be used and where phonondislocation interaction has to be taken into account. Such a theory for planar rotator system in $2 \mathrm{D}$ which is a similar problem has been developed by one of the authors. ${ }^{17}$ Without going into detail we wish to present only one interesting result of such a theory. Assume that one neglects dislocation-phonon interaction in the liquid state then one has essentially a gas of interacting dislocations. The canonical pressure $p$ of that gas of dislocations is given by

$$
p v_{d}=k_{B} T\left(1-\frac{1}{4} \beta q^{2}\right),
$$

where $q^{2} \equiv K b^{2} / 4 \pi$ and $v_{d}$ was defined earlier. This equation holds for $k_{B} T>\frac{1}{2} q^{2}$ and $\sum_{\alpha} \vec{b}^{\alpha}=0$, and is the same as the one obtained for the two-component Coulomb plasma. ${ }^{18}$

\section{QUALITATIVE THEORY OF FIRST- AND SECOND- ORDER MELTING PHASE TRANSITION}

Although it is well known that in $3 \mathrm{D}$ systems so far only discontinuous melting PT from crystalline solids to liquids have been observed there exist examples of continuous and discontinuous PT in two-dimensions. We will study this problem using the theory developed in Secs. II and III and in addition already existing arguments based on the droplet model of condensation. ${ }^{19,20}$

Consider first the molecular-dynamics computer experiments of Hockney and Brown. ${ }^{6}$ Here classical point particles confined to move in the plane interact with each other via a $1 / r$ potential. There is also a charge compensating background present which renders the system stable. Such a system orders in a triangular lattice. It can now be seen from Fig. 4(a) of Ref. 6 that the ground state of that system at $T \neq 0$ is a polycrystalline phase. As a matter of fact it consists of two types of domains 
going over into each other by $30^{\circ}$ rotation. Loss of long-range directional order occurs via thermal production of more and more domains and eventually by a $\lambda$-type PT into a state with a distribution function characteristic of a liquid. It also follows from Fig. 4(a) of Ref. 6 that dislocations are produced in pairs as $T$ increases and that there is a considerable increase of the vibrational amplitude when the system approaches the PT. The novel aspect of this problem is therefore the thermal nucleation of domains and the domain boundary dynamics. In contrast to dislocations domain boundaries in 2D crystals have to be considered as 1D topological objects. It is well known that thermal nucleation or propagation of domain boundaries in three dimensions requires too large activation energies as compared to thermal nucleation of $1 \mathrm{D}$ dislocation loops. It is therefore very suggestive that in cases where $(D-1)$-dimensional topological objects are possible energetically continuous melting transition may occur. In the following a qualitative theory for that problem will be given.

For 2D systems with long-range $1 / r$ interaction it has been shown by Meissner et al..$^{21}$ that the triangular lattice has a stable phonon excitation spectrum. In the harmonic approximation for a $1 / r^{\alpha}$-interaction with $0<\alpha<2$ one develops the lattice deviations around the triangular lattice equilibrium positions. This gives the potential part of the Hamiltonian

$$
\begin{aligned}
H^{(2)}=\frac{1}{2} \alpha(\pi n)^{\alpha / 2} e^{2} \sum_{\substack{i, j \\
i \neq j}} \frac{1}{\left|\overrightarrow{\mathrm{r}}_{i}-\overrightarrow{\mathrm{r}}_{j}\right|^{\alpha+2}} \\
\times\left\{-\left(\overrightarrow{\mathrm{u}}_{i}-\overrightarrow{\mathrm{u}}_{j}\right)^{2}+(\alpha+2)\right. \\
\left.\quad \times\left[\left(\overrightarrow{\mathrm{u}}_{i}-\overrightarrow{\mathrm{u}}_{j}\right) \cdot \overrightarrow{\mathrm{n}}_{i-j}\right]^{2}\right\},
\end{aligned}
$$

where $\vec{u}_{i}$ is the $i$ th lattice point deviation from its equilibrium position $\overrightarrow{\mathrm{r}}_{i}$, and $\overrightarrow{\mathrm{n}}_{i-j} \equiv\left(\overrightarrow{\mathrm{r}}_{i}-\overrightarrow{\mathrm{r}}_{j}\right) /\left|\overrightarrow{\mathrm{r}}_{i}-\overrightarrow{\mathrm{r}}_{j}\right|$, $n$ is the density of particles, $(\pi n)^{\alpha / 2} e^{2}$ has dimension of energy, and all other quantities are dimensionless. The interaction with the charge compensating background can be neglected as long as one is not concerned with the $q=0$ mode of the system. The phonon dispersion is obtained by diagonalizing the inverse phonon propagator

$$
\begin{aligned}
\left(\Delta_{\overrightarrow{\mathrm{q}}}\right)_{i j}=\sum_{s \neq 0} & \frac{1}{r_{s}^{\alpha+2}}\left(1-\cos \overrightarrow{\mathrm{q}} \cdot \overrightarrow{\mathrm{r}}_{s}\right) \\
& \times\left[-\delta_{i j}+(\alpha+2) n_{\left.r_{s, i} n_{r_{s, j}}\right],}\right.
\end{aligned}
$$

where $i, j$ ran over the two coordinates of the plane. The interaction between dislocations is governed by the Green's-function tensor

$G\left(\overrightarrow{\mathrm{r}}-\overrightarrow{\mathrm{r}}^{\prime}\right)=\frac{1}{(2 \pi)^{2}} \int d^{2} q \Delta_{\overrightarrow{\mathrm{q}}}^{-1}\left[1-\cos \overrightarrow{\mathrm{q}} \cdot\left(\overrightarrow{\mathrm{r}}-\overrightarrow{\mathrm{r}}^{\prime}\right)\right]$, where $q$ integration is done over the first Brillouin zone of area $(2 \pi)^{2}$. From dimensionality arguments it follows now that Eq. (31) allows longitudinal phonons with dispersion $\omega_{q}^{l} \sim q^{\alpha / 2}$ for $q \ll 1$. Applying the Ewald summation method to Eq. (31) (see, e.g., Meissner et $a l .{ }^{21}$ ) it can be shown that the transversal phonon branch has linear dispersion $\omega_{q}^{t} \sim q$, $q \ll 1$. From this follows that for $0<\alpha<2$ no longrange positional order is possible. The same applies to $\alpha \geq 2$ only that there $\omega_{q}^{\gamma} \sim q$. For $\alpha=0$ one uses a $\log _{10} r$ interaction which is the Coulomb interaction in two dimensions and where the longitudinal mode shows the plasma gap but $\omega_{q}^{t} \sim q$ still holds. It follows from the linearity of $\omega_{q}^{t}$ in $q$ for $q \ll 1$ that the leading term given by Eq. (32) which governs the dislocation interaction depends logarithmically on the distance as it is the case for short-range interaction. The earlier developed theory for dislocations therefore applies qualitatively in the same fashion. Concerning now the production of $30^{\circ}$ domain boundaries in a triangular lattice one observes the following. From symmetry follows that for a triangular lattice rotation by $30^{\circ}$ of the center of a domain compromising all neighbors up to $n$th order may lead to metastable states. In order to estimate the energy $\delta E$ of such a domain we calculate the interaction energy of a $30^{\circ}$ rotated circular domain of radius $r$ with the rest of the crystal. The idea is essentially the same as used in Refs. 19, 20 in another context. Because we do not consider macroscopic charge density perturbation the uniform background does not enter. In order to obtain the internal energy of the $30^{\circ}$ rotated domain one moves each lattice point of the original lattice into the position of the closest lattice point of the rotated system. In this way no macroscopic material transport occurs. Accordingly the magnitude of the deviations $\vec{u}_{i}$ of the rotated domain must be a fraction, say $\delta_{0}$, of the interatomic distance. From this prescription it follows that the $\vec{u}_{i}$ 's in Eq. (30) belonging to the circular domain can be averaged over all angles and that only interactions in Eq. (30) between points inside and outside the circular domain have to be taken into account. After some simple algebra one obtains for $\alpha=1$

$$
\delta E / k_{B} T \sim \delta_{0}^{2} \Gamma_{1} n_{r},
$$

where $\Gamma_{1} \equiv \pi^{1 / 2} n^{1 / 2} e^{2} / k_{B} T$, and $n_{r}$ is the number of lattice points on the boundary of the circular domain. Equation (33) shows that the interaction energy for $\alpha=1$ between the domain rotated by $30^{\circ}$ with respect to the rest of the crystal can be expressed as a boundary energy. In case that Eq. (33) holds down to sufficiently small crystallites and arbitrary circumference the configurational free energy associated with the polycrystalline domains 
can be calculated via a two-state Ising model. Although Eq. (33) follows simply from dimensional arguments it is not so simple to determine the proportionality constant to Eq. (33). As this requires rather specific assumptions about the lattice displacement in the boundary we are presently not in a position to determine the transition temperature from the Ising model. Our interpretation of this PT is that there are two disordering mechanisms. One is the production of dislocations in pairs and the other is the generation of domain boundaries. The first mechanism tries to produce a discontinuous PT and the second a continuous PT. We expect that the domain boundaries affect the elastic dipole interaction. The discontinuous condensation phenomena for the elastic dipoles predicted in Sec. III may therefore be smoothed out because interaction between dipoles may be confined to one crystallite. The latent heat going into the first-order PT driven by the elastic dipoles alone may therefore lead to a steepening of the specific heat anomaly, i.e., the $\log$ anomaly of the Ising model may go over into a power law singularity as it is observed by Hockney and $B$ rown ${ }^{6}$. Let us point out that in phase diagrams where lines of second-order PT go over into lines of first-order PT at a tricritical point $T_{3 c}$ mean-field theory predicts at $T_{3 c}$ a specificheat exponent $\alpha=\frac{1}{2}$ in three-dimensions. In general therefore a considerable steepening of the specific heat anomaly can be expected if a system is on the brink to change its state discontinuously.

For general $\alpha$ one obtains instead of Eq. (33)

$$
\delta E / k_{B} T\left\{\begin{array}{l}
\sim \delta_{0}^{2} \Gamma_{\alpha} n_{c}^{(2-\alpha) / 2}, \quad 0<\alpha<2, \\
\sim \delta_{0}^{2} \Gamma_{0} n_{c} \ln N, \quad \alpha=0 .
\end{array}\right.
$$

Here $\Gamma_{\alpha} \equiv(\pi n)^{\alpha / 2} e^{2} / k_{B} T, n_{c}$ is the number of lattice points in a $30^{\circ}$ rotated circular domain and $N \ll n_{c}$ the number of lattice points of the total system. The proportionality constants to Eqs. (34a) and (34b) have not been calculated. It follows from Eq. (34b) that thermal nucleation of domains for $\alpha=0$ is prohibited above a certain critical $\Gamma_{0}^{*}$ for which the change of free energy

$$
\delta F / k_{B} T \sim\left(f \delta_{0}^{2} \Gamma_{0} n_{c}^{*}-1\right) \ln N
$$

is positive. In Eq. (35) $f$ is a numerical factor and $n_{c}^{*}$ is the smallest number of lattice points in a circular domain for which the model still makes sense, i.e., $n_{c}^{*}$ should hold at least the points of the nearest- and next-nearest-neighbor circles. The argument to derive Eq. (35) is the same as the one Kosterlitz and Thouless use for dislocations. The second term of Eq. (35) originates from the entropy of the considered domain. For $\Gamma_{0}<\Gamma_{0}^{*}$ the system is thermodynamically unstable against domain formation and will thus disorder. However, because elastic dipole nucleation occurs already for $\Gamma_{0}>\Gamma_{0}^{*}$ it can be expected that the melting PT is essentially driven by the elastic dipoles. In any case the PT will be discontinuous for $\alpha=0$, because both mechanisms mentioned will lead to a discontinuous PT.

Unfortunately there are no molecular dynamics experiments with $\log$ potentials $(\alpha=0)$ in two-dimensions to confirm these results. However, for the $1 / r$ potential which in three dimensions is the analogous problem one knows from the computer experiments of Pollock and Hansen ${ }^{22}$ that at $\Gamma_{1}^{c}$ $=\left(\frac{4}{3} \pi n\right)^{1 / 3} e^{2} / k_{B} T=155 \pm 10$ a discontinuous melting PT occurs. Going through the same arugments which led to Eqs. (34a) and (34b) one obtains for a $1 / r^{\alpha}$ potential for the domain energy in three dimensions

$$
\delta E / k_{B} T\left\{\begin{array}{l}
\sim \delta_{0}^{2} \Gamma_{\alpha} n_{s}^{(4-\alpha) / 3}, \quad 1<\alpha<4 \\
\sim \delta_{0}^{2} \Gamma_{1} n_{s} \ln N, \quad \alpha=1
\end{array}\right.
$$

Here $\Gamma_{\alpha} \equiv\left(\frac{4}{3} \pi n\right)^{\alpha / 3} e^{2} / k_{B} T, n_{s}$ the number of points in a metastable spherical domain and $N$ the number of points in the system. Using the same arguments as below Eq. (35) it follows that for $\alpha=1$ a discontinuous PT has to occur in accordance with Pollock and Hansen. ${ }^{22}$ Furthermore for Eq. (36a) it follows that for $\alpha=2$ the analogous situation arises as for $\alpha=1$ in two dimensions because then $\delta E / k_{B} T$ is proportional to the surface of the domains. Depending now on the number $n_{m}$ of different metastable domains in three dimensions for the $1 / r^{2}$ potential an $n_{m}$-state Ising model may describe the configurational free energy associated with the domain structure. Accordingly we conjecture that the $1 / r^{2}$ potential in three dimensions may lead to continuous melting PT, if the $n_{m}$-state Ising model belonging to the stable crystal structure of the $1 / r^{2}$ potential allows a continuous PT.

One can also check these ideas against the results of the corresponding one-dimensional problem. Here one knows that for the Coulomb potential $r$ the system is at all temperatures in an ordered state. ${ }^{23}$ Because this system does not allow topological defects, i.e., there are no possible metastable domains and nothing what corresponds to dislocations there exists also nothing which could invalidate simple perturbation theory as the temperature arises. The point is that the topological defects or metastable states occuring in two and higher-dimensions enter through the anharmonic lattice potential where they cannot be taken care of by perturbation theory.

Finally we would like to point out that in systems with short-range interaction similar ideas as those developed above may apply. In cases where the 
chemical potential for domain formation is small compared to the chemical potential for elastic dipole formation a continuous melting PT may arise. In the opposite case the PT is mainly driven by the elastic dipole dissociation and therefore is discontinuous as argued in Sec. III. Because in real systems with hard cores the sytem is not characterized by just one $\Gamma$ as for the systems considered above rather interesting phase diagrams over the coupling constant space may arise. We expect, however, that with increasing size of the constituent particles geometrical effects will produce rather large boundary energy and rather large activation energy for domain nucleation and domain boundary propagation. Intuitively one believes also that the lower-dimensional topological defects will be more favorable energetically. We expect that in cases where the melting PT is continuous rather large deviations from the critical properties of the reference problem discribing the configurational free energy of the polydomain system may arise.

In concluding this section we would like to make some additional remarks which respect to the $30^{\circ}$ rotated domains which we have introduced rather heuristically. In that context we like to point out first that the theory of dislocations and metastable states in general can be approached by similar methods as developed by Berezinskii ${ }^{1}$ for the metastable states of the planar rotator model. In this approach the phase space of the problem is subdivided into sectors, where the sectors correspond to metastable states. In order to introduce dislocations in this formalism one introduces a set of vectors $\left\{\vec{c}_{i j}\right\}$ for each nearest neighbor bond, where $\overrightarrow{\mathrm{c}}_{i j}$ is a lattice vector and $\overrightarrow{\mathrm{c}}_{i j}=-\overrightarrow{\mathrm{c}}_{i j}$ holds. The Hamiltonian corresponding to a sector of the phase space characterized by $\left\{\overrightarrow{\mathrm{c}}_{\boldsymbol{i}}\right\}$ is then given by

$$
H\left(\left\{\overrightarrow{\mathrm{c}}_{i j}\right\}\right)=\sum_{\langle i, j\rangle} v\left(\overrightarrow{\mathrm{u}}_{i}-\overrightarrow{\mathrm{u}}_{j}-\overrightarrow{\mathrm{c}}_{i j}\right),
$$

where $v(\overrightarrow{\mathrm{x}})$ represents a potential which for sake of simplicity we restrict to nearest-neighbor interaction. Furthermore $\vec{u}_{i}$ is the displacement of the $i$ th lattice constituent from its presumed equilibrium position in the ground state of the system, where $\left\{\vec{c}_{i j}\right\} \equiv 0$. One defines next a Burgers vector for each lattice cell $\alpha$ by means of $\vec{b}^{\alpha}$ $=\sum \vec{c}_{i j}$, where the contour is taken counterclockwise around the $\alpha$-th cell. Diagonalizing Eq. (37) around its equilibrium position which is a function of the set $\left\{\overrightarrow{\mathbf{c}}_{\boldsymbol{i}}\right\}$ one obtains the interaction law for dislocations. For sake of simplicity one may do this using $v(\overrightarrow{\mathrm{x}}) \sim x^{2}$. Using an anharmonic potential $v(\overrightarrow{\mathrm{x}})$ allows then in a natural way to introduce phonon-dislocation interaction.

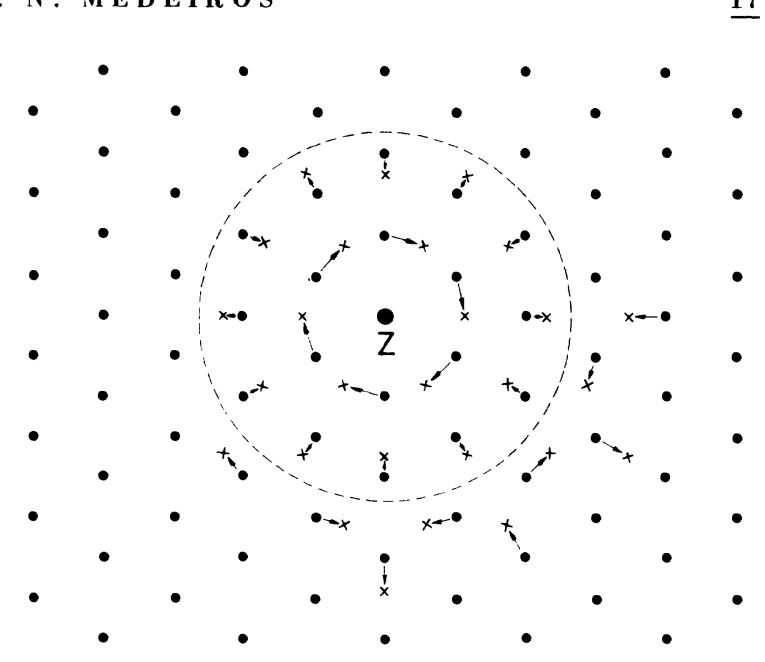

FIG. 3. Schematic illustration of $30^{\circ}$-rotated domain bounded by dashed circle in a triangular lattice.

In a similar way the $30^{\circ}$ rotated domains for a triangular lattice may be introduced. For sake of simplicity we describe this procedure graphically by means of Fig. 3. The points in Fig. 3 corresponds to an ideal triangular lattice and the crosses to a triangular lattice rotated aroung the center point $Z$ by $30^{\circ}$. The arrows describe the path a lattice constituent moves from its position in the ideal triangular lattice to its position in the $30^{\circ}$-rotated domain. Consider first the domain inside the dashed circle and ignore the crosses drawn outside of this circle. It is then obvious from visual inspection that the lattice constituents on either side of the dashed circle will relax only slightly from their indicated positions. This is a consequence of the high symmetry of the arrangement. It is also a simple matter to convince oneself from the metastability of the arrangement by using instead of point-like lattice constituents particles with a finite core diameter. The energy and configuration of that metastable state can now be calculated in principle but has not been done as yet. It follows also from Fig. 3 that forming a $30^{\circ}$-rotated domain using nearest neighbors to the point $Z$ only leads to an unstable configuration. We think therefore that the metastable domain with the dashed circle as circumference has lowest internal energy. Accordingly thermal nucleation of metastable $30^{\circ}$ domains will start with the one shown in Fig. 3. Growth of such a domain will lead over activation barriers into metastable states of higher internal energy. Intuitively one expects that for short-range forces growth of the domain will not produce macroscopic strain and that therefore the internal energy increases proportional to its circumference. This, of course, excludes pathologically shaped circumferences. The fact that for long-range repulsive interaction between 
point particles and a charge compensating background a similar result holds for $\alpha=1$ [according to Eq. (33) and Eq. (34a)] is not immediately obvious. Finally we like to point out that there are perhaps other metastable states besides the $30^{\circ}$ rotated domains. It is clear, however, that misorientated domains have to come into existence over a nucleation mechanism starting with smaller-sized domains otherwise their formation probability will be insignificantly small. The metastable domain shown in Fig. 3 seems to involve the smallest number of lattice constituents to be displaced appreciably besides the dislocation pairs. It is reasonable therefore to assume that its formation will be energetically most favorable besides the formation of dislocation pairs. Accordingly also larger-sized $30^{\circ}$ domains which come into existence over a growth mechanism can be expected besides dislocation pairs with highest probability.

\section{DISCUSSION OF MONOLAYER MELTING TRANSITION}

In this section we would like to make an interpretation of various solid-liquid-type PT observed in monolayer systems adsorbed on graphite in terms of the theory developed. One of the problematic features such interpretational work faces is that the substrate potential enters the problem and that there are vertical degrees of freedom. The latter point is usually not very serious for sufficiently strong adsorption. The periodical potential of the graphite substrate, however, may confine the adsorbed monolayer to epitaxy and thus to true long-range positional order. This effect can be taken into account by providing the monolayer lattice modes with a mass, i.e., $\omega_{q}^{l, t^{2}} \sim m_{l, t}^{2}$ $+c_{l, t}^{2} q^{2}$, where the ratio $m_{l} / c_{l}$ or $m_{t} / c_{t}$ is a measure for the strength of adsorbing forces to monolayer forces. Because the dislocation interaction is determined by Eq. (32) where the phonon spectrum enters, the finite masses $m_{l}$ and $m_{t}$ will qualitatively change the interaction into a finiteranged interaction. This means that the $\log _{10} r$ interaction between dislocations will be screened off for $r>1 / m_{t}$. This implies that dislocation pairs may dissociate at any finite $T$ as it is observed for molecules bound by ordinary forces. This effect will certainly depend on the order of magnitude of $m_{t} / c_{t}$. For $m_{t} / c_{t} \gg 1$ the system is presumably better studied using a static orderdisorder model, whereas for $m_{t} / c_{t} \ll 1$ the monolayer lattice modes will be important and the theory as developed here is more appropriate. We like to point out that in cases where the size of the adatoms is such that a commensurate phase with the substrate is not possible due to geometrical constraints one should have in the ideal case $m_{t(l)} / c_{t(l)}=0$. Only in the case where defects of the substrate are present or there is an elastic response of the substrate $m_{t(l)} / c_{t(l)} \neq 0$ is possible. It is clear, however, that also in the case of a nonregistered phase the substrate potential exerts a force field on the monolayer. Linearizing this force field around the presumed equilibrium configuration of the nonregistered monolayer, however, is problematic because of $\left\langle[\overrightarrow{\mathrm{u}}(\overrightarrow{\mathrm{R}})]^{2}\right\rangle \sim \ln N$. For finite-sized monolayers therefore the approximation will work, but not for infinitely extended nonregistered monolayers. In the latter case only quantities depending on the relative deviations $\left[\overrightarrow{\mathrm{u}}(\overrightarrow{\mathrm{R}})-\overrightarrow{\mathrm{u}}\left(\overrightarrow{\mathrm{R}}^{\prime}\right)\right]$ can be expanded. ${ }^{1}$

In cases where the geometrical size of the adatoms allows a registered phase and where the substrate potential holds locally bound states a crystalline ordered state is possible at low $T$. If in cases where $m_{t} / c_{t} \ll 1$ besides the usual orderdisorder PT at $T_{N}$ where the system loses longrange order a topological type of PT occurs is not quite clear. We are presently not in a position to decide this question. It is clear that for $m_{t} / c_{t} \neq 0$ the dislocation pairs will dissociate with increasing $T$ and in this way accomplish disordering. Besides this mechanism there exists, however, still the possibility of a density type of PT at $T_{c}\left(n_{d}\right)$ where disordering is accomplished discontinuously as it is the case for $m_{t} / c_{t}=0$. In the following we propose that this is the case for the registered phase of the $\mathrm{N}_{2}$ monolayer on graphite.

It should also be pointed out that order-disorder transitions in monolayers may be continuous or discontinuous. A classification of order-disorder transitions in commensurate monolayer systems has been given by Domany et al. ${ }^{24}$ We like to mention in this context that a lattice gas model is presumably most appropriate for structures which are rather loosely packed. In addition the substrate potential should be rather strong so that kinetic effects are of no importance. In more densely packed commensurate adsorbed systems defects in the ordered state may produce rather far reaching disturbances in the structure which are hard to assess in a short-range interacting lattice gas model. The interaction between defects over appreciable distances depending on the ratio $m_{t(l)} / c_{t(l)}$ may change the qualitative nature of the problem. In the following we will discuss a number of experiments in the light of the ideas exposed here.

Consider first the experiment of Schechter, Suzanne, and Dash ${ }^{8}$ where a discontinuous melting PT of 1-3, butadiene iron tricarbonyl (BIT), 
$\mathrm{C}_{4} \mathrm{H}_{6} \mathrm{Fe}(\mathrm{CO})_{3}$ adsorbed on graphite has been observed. A discontinuous PT has been observed for three different coverages $\times=0.33,0.63$, and 1.04. The effective molecular area $41 \AA^{2}$ corresponds to $X=1$. Due to the rather large molecular extensions of BIT we can assume that the periodic substrate potential plays no role. From the discontinuous nature of this PT we conclude that it is described by the dissociation of elastic dipoles as explained in Sec. III. Presumably the monolayer has a trigonal structure, but thermal nucleation of $30^{\circ}$-rotated domains is prohibited on account of too large formation energy. We do not have an explanation of the decrease of melting temperature $T_{M}$ with increasing $x$ as exhibited by Fig. 2 of Ref. 8.

As a second example we consider the $\mathrm{N}_{2}$ monolayer work of Kjems et al. ${ }^{7}$ Here the authors have evidence that the low-density phase forms epitaxially on the graphite surface in the $\sqrt{3} \times \sqrt{3}$ structure. Accordingly each third of the graphite hexagonal potential wells is occupied by a $\mathrm{N}_{2}$ molecule. The nearest-neighbor distance of the occupied centers of the hexagonal potential wells is $4.26 \AA .^{25}$ The extensions of the $\mathrm{N}_{2}$ molecule are given in Ref. 26 by 4.36 and $3.38 \AA$ for length and width, respectively. It follows from this that walls between domains belonging to one of the three possible translated structures arising from the $x=\frac{1}{3}$ coverage must have fairly high energy due to geometrical constraints. Furthermore due to epitaxy $30^{\circ}$-rotated domains are energetically unfavorable because their energy increases with the domain size. Formation of dislocation pairs, however, is possible. We suggest therefore that the discontinuous PT to a presumably liquid phase as observed by Kjems et al. ${ }^{7}$ may be due to a dissociation of elastic dipoles as argued in Sec. III. It is understood, however, that the $\log _{10} r$ interaction in commensurate phases is screened due to $m_{t} / c_{t} \neq 0$. Accordingly we cannot make a definite statement about the nature of the transition as has been pointed out earlier. Due to the geometrical constraints produced by the rather large $\mathrm{N}_{2}$ molecules the static order-disorder aspect of the problem where each $\mathrm{N}_{2}$ molecule hops from one hexagonal well to another for entropy production is presumably not realistic if longer-ranged disturbances are not taken into account. In addition it has to be shown that the states used in a lattice gas description a re really metastable. The orderdisorder transition for a $x=\frac{1}{3}$ coverage should also be continuous if the three-state Potts model would apply. Furthermore Kjems et al. ${ }^{7}$ have observed a denser phase where the melting transition is continuous. Because this structure is not constrained by epitaxy $30^{\circ}$-rotated domains are en- ergetically possible and we propose therefore that the continuous nature of this PT arises from the effect explained in Sec. IV. In the present case, however, the configurational free energy should be described by the two-state Ising model. It would be interesting in that context to have experimental evidence on the domain structure of such monolayers. It would also be interesting to know the order of the melting PT of ${ }^{36} \mathrm{Ar}$ monolayers which form a nonregistered phase ${ }^{27}$ It follows from the existence of nonregistered monolayer phases on graphite that interatomic forces in the monolayers must play the dominant part in determining the monolayer structure. ${ }^{7}$ This implies that the conditions for applying an ideal monolayer theory are well satisfied.

As a third example we consider the PT observed in ${ }^{3} \mathrm{He}$ and ${ }^{4} \mathrm{He}$ monolayers on graphite. In a recent experiment $\mathrm{Bretz}^{9}$ has measured the ordering transition in ${ }^{3} \mathrm{He}$ and ${ }^{4} \mathrm{He}$ on high-quality graphite substrate. He observes a rather strong specificheat anomaly with critical exponent $\alpha \sim 0.36$. This critical-heat exponent is substantially larger as Bretz $^{9}$ emphasizes as the $\alpha$ expected from the static three-state Potts model as proposed by Alexander ${ }^{28}$ for this system. Although quantum effects do not dominate frequently critical behavior one expects here that the rather large zeropoint motion and the hexagonal net of weak potential wells on graphite substrate gives the lowlying excitation modes of the ordered monolayers a phonon like character. We suggest considering the ordered phase of ${ }^{3} \mathrm{He}$ and ${ }^{4} \mathrm{He}$ as a $2 \mathrm{D}$ solid with three different types of domains. The latter can be obtained from a given domain by translation along one of the nearest-neighbor vectors connecting the centers of the graphite hexagonal wells. In addition there exists the possibility for dislocations and other defects to come into existence in an ordered domain. Due to the smaller size of the He atoms we expect that the problem allows three types of domains in contrast to epitaxial $\mathrm{N}_{2}$ layers. Accordingly the configurational free energy associated with the network of crystallite boundaries can be described by a three-state Potts model. ${ }^{30}$ Because this model cannot hold down to smallest crystallites and because there are also dislocations produced in pairs, and there are phonon vibrations coupled to the metastable states above, it follows that the continuous PT of the Potts model predicted by Baxter ${ }^{31}$ will be modified. We expect this modification to be similar to the one discussed in Sec. IV for the point particle system studied by Hockney and Brown. ${ }^{6}$ In particular we think that the additional activation of degrees of freedom associated with the elastic dipoles will produce a steepening of the specific- 
heat anomaly of the pure Potts model as it is observed. ${ }^{9}$ We have not studied the effect of quantum tunneling ${ }^{29}$ on dislocation formation and propagation. We expect, however, that the generation of topological defects is facilitated by such effects because the usual activation energy involved is reduced.

With respect to numerical estimates of the transition temperature $T_{c}\left(n_{d}\right)$ (where $n_{d}$ is determined by $2 \mu$ ) for specific monolayer systems where melting occurs via a dissociation of elastic dipoles a crude estimate can be obtained from $T_{c}\left(n_{d}\right)<T_{M}^{*}$. This requires, however, that $K$ is known which in principle can be obtained via the phonon spectrum. It is clear, however, that the anisotropy of real lattice systems will modify the upper bound to $T$, i.e., $T_{M}^{*}$ from its value in the isotropic system appreciably. This has been shown in Ref. 17 for planar rotator systems, where the analogous quantity to $T_{M}^{*}$ differs for square and triangular lattices by a factor of $1 / \sqrt{3}$. It follows from this that under the present circumstances we are not in a position to give a quantitative justification of the fairly qualitative theory developed.

Let us point out that our theory in contrast to the theory of Kosterlitz and Thouless ${ }^{2}$ leads to a discontinuous melting transition in two dimensions when driven by dislocations. In principle it is now possible to incorporate into our formalism the polarization effects of the dipole gas on the self-energy of one dipole as it has been done in Ref. 2. It is possible that this will produce some modification of the theory developed which is essentially based on physical concepts familiar from 3D physics. We do not think, however, that a more sophisticated theory will change the nature of the melting transition obtained by us. The theory of Ref. 2 implies softening of the longwavelength transversal phonon branch to zero at the melting temperature. Such effects have often been postulated for three dimensions but failed on experiment. Because the dissociation of elastic dipoles can occur continuously or discontinuously the problem of the order of the PT cannot be decided by means of a symmetry argument but is a problem of stability. We propose that the stability properties of the model of Ref. 2 should also be studied, i.e., the statistical mechanics of the spatial dipole distribution functions should be incorporated in the model. The latter distribution furctions are treated by the authors of Ref. 2 purely stochastically. Because their melting transition leads smoothly into the liquid state where the dislocation distribution functions are not stochastic it is obvious that this point has to be modified.

In concluding this section we would like to consider briefly melting in three dimensions. From the extensive molecular-dynamics experiments of Cotterill et al..$^{32}$ using a truncated Lennard-Jones potential it follows that the melting process there is initiated by the generation of tiny dislocation loops of the Schockley type (see, e.g., Hirth and Lothe ${ }^{33}$ ). The self-energy of such loops is $\sim r \ln r$ if they are circular ${ }^{33}$ and of radius $r$ and when they are in the dipole configuration their self-energy is $\sim l$ lnd, where $l$ is the length and $d$ is the width of the elongated loop. The latter configuration is presumably energetically more favorable and has higher elastic polarizability as the circular loop. Because the loops like other defects have finite internal energy they will be present at all temperatures. If they do not interact with each other they will never produce a PT when $T$ increases. Interaction, however, between the loops may produce a PT. It follows from Figs. 4-6 of Ref. 32 that initial overlap effects between tiny loops are not present. Accordingly we propose that the discontinuous melting PT observed is also here a consequence of a condensation phenomena due to interaction between metastable objects. In contrast to the theory of two-dimensional melting developed in Secs. II and III the topological defects are here one-dimensional loops. That the elastic polarizability of the loops does not define a finite temperature $T_{M}^{*}$ where it diverges due to the $r \ln r$ dependence is irrelevant. The main point is that the $r \ln r$ or $l \ln d$ self-energy does not allow the loops to disintegrate which would open the possibility to get steadily from the crystalline to the liquid phase. Such a transition could be compared to a continuous insulator-semiconductormetal transition with increasing $T$ in a system where the band gap is produced by the lattice potential. The semiconducting properties of the latter system would compare to thermal plasticity properties of the mechanical system when loops are allowed to get infinitely large sized.

The main point of our argument is that also in three dimensions the discontinuous nature of the melting PT is a consequence of the interaction between metastable states which leads to a gasplasma-type PT and in this way achieves the melting of the solid. There is little overlap between the topological objects involved in the gas-plasma transition when the process is initiated. Accordingly also here the approach to the problem by neglecting these effects altogether in lowest approximation as we did in Secs. II and III may be justified. Similar as in Eq. (20) there exists a critical density $n_{l}(T)$ of loops for which $K^{\prime}=0$ and a critical density $n_{l}^{c}(T)<n_{l}(T)$ where a condensation phenomena occurs leading presumably into the state $K^{\prime}=0$.

In three-dimension thermal nucleation of domain 
boundaries is of no importance because it involves too many atoms and therefore leads to too high activation energies. Melting is therefore always discontinuous and driven by a dislocation mechanism. The gas-plasma-type PT picture leading to a discontinuous transition should therefore essentially be the correct one to describe melting in three dimensions. In addition it supports the theoretical approach for two dimensions where due to the configurational effects of domain boundaries continuous PT occur and in this way direct experimental verification of the theory is prevented. In $3 \mathrm{D}$ cases where appreciable effects from configurational free energy associated with a thermally nucleated polycrystalline structure are present as discussed for the $1 / r^{2}$-potential in Sec. IV a new theory has to be worked out. This problem and the development of an analogous theory in three dimensions to the one developed in Secs. II and III for two dimensions is presently in progress.

\section{ACKNOWLEDGMENTS}

One of the authors ${ }^{-}$(A.H.) stay at the Instituto de Fisica of Universidade Federal do Rio Grande do Sul has been arranged within the agreement DAAD (Germany)-CNPq (Brazil). A.H. is also grateful to W. Helfrich for interesting discussions on the problem.
*Work supported in part by Deutsche Forschungsgemeinschaft SFB 161 .

${ }^{1}$ V. L. Berezinskii, Sov. Phys.-JETP $\underline{32}, 493$ (1971); $\underline{34}$, 610 (1972).

${ }^{2}$ J. M. Kosterlitz and D. J. Thouless, J. Phys. C $\underline{6}, 1181$ (1973).

${ }^{3}$ V. N. Popov, Sov. Phys.-JETP 37, 341 (1973).

${ }^{4}$ N. D. Mermin, Phys. Rev. 176, 250 (1968).

${ }^{5}$ D. Kuhlmann-Wilsdorf, Phys. Rev. 140, A1599 (1965).

${ }^{6}$ R. W. Hockney and T. R. Brown, J. Phys. C 8,1813 (1975).

${ }^{7}$ J. K. Kjems, L. Passell, H. Taub, J. G. Dash, and A. D. Novaco, Phys. Rev. B 13, 1446 (1976).

${ }^{8}$ H. Schechter, J. Suzanne, and J. G. Dash, Phys. Rev. Lett. 37, 706 (1976).

${ }^{9}$ M. Bretz, Phys. Rev. Lett. 38, 501 (1977).

${ }^{10} \mathrm{~A}$. Holz, Fourth MECO Conference in Uterägeri Switzerland, April, 1977 (unpublished).

${ }^{11}$ A. Seeger, Phys. Status Solidi 1, 669 (1961).

${ }^{12} \mathrm{~J}$. Friedel, in Dislocations (Pérgamon, London, 1967), Chap. II.

${ }^{13} \mathrm{~J}$. De Boer, F. van der Maesen, and C. A. ten Seldam, Physica (Utr.) 19, 265 (1953).

${ }^{14}$ W. F. Brown, in Encyclopedia of Physics, edited by S. Flü gge (Springer-Verlag, Heidelberg, 1956), Vol. 17 , p. 1 .

${ }^{15}$ L. Onsager, J. Am. Chem. Soc. 58, 1486 (1936).

${ }^{16}$ A. Holz and K. H. Bennemann, Phys. Rev. Lett. 37, 1507 (1976).
${ }^{17}$ A. Holz (unpublished).

${ }^{18} \mathrm{C}$. Deutsch and M. Lavaud, Phys. Lett. A $\underline{39}, 253$ (1972).

${ }^{19}$ M. E. Fisher, J. Appl. Phys. 38, 981 (1966).

${ }^{20}$ N. D. Mermin, J. Phys. Soc. Jpn. Suppl. 26, 203 (1969).

${ }^{21} \mathrm{G}$. Meissner, H. Namaizawa, and M. Voss, Phys. Rev. B 13, 1370 (1976).

${ }^{22}$ E. L. Pollock and J. P. Hansen, Phys. Rev. A 8, 3110 (1973).

${ }^{23}$ H. Kunz, Ann. Phys. (N. Y.) 85, 303 (1974).

${ }^{24}$ E. Domany, M. Schick, and J. S. Walk, Phys. Rev. Lett. 38, 1148 (1977).

${ }^{25}$ J. Suzanne, J. P. Coulomb, and M. Bienfait, Surf. Sci. 40, 414 (1973)

${ }^{26}$ A. Bourdon, C. Marti, and P. Thorel, Phys. Rev. Lett. 35, 544 (1975).

${ }^{27}$ H. Taub, L. Passell, J. K. Kjems, K. Carneiro, J. P. McTague, and J. G. Dash, Phys. Rev. Lett. 34, 654 (1975).

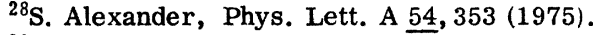

${ }^{29}$ B. P. Cowan, M. G. Richards, A. L. Thomson, and W. J. Mullin, Phys. Rev. Lett. 38, 165 (1977).

${ }^{30}$ R. B. Potts, Proc. Camb. Philos. Soc. 48. 106 (1952).

${ }^{31}$ R. J. Baxter, J. Phys. C $\underline{6}$, L445 (1973).

${ }^{32}$ R. M. J. Cotterill, W. Damgaard Kristensen, and E. J. Jensen, Philos. Mag. 30, 245 (1974).

${ }^{33} \mathrm{~J}$. P. Hirth and J. Lothe, Theory of Dislocations (McGraw-Hill, New York, 1968). 